\title{
First-line treatment for patients with advanced non-small cell lung carcinoma and high PD-L1 expression: pembrolizumab or pembrolizumab plus chemotherapy
}

\author{
Yixin Zhou ${ }^{1,2,3 \dagger}$, Zuan Lin ${ }^{1,2,4 \dagger}$, Xuanye Zhang ${ }^{1,2,5 \dagger}$, Chen Chen ${ }^{1,2,6}$, Hongyun Zhao ${ }^{1,2,4}$, Shaodong Hong ${ }^{1,2,5^{*}}$ (D) and \\ Li Zhang ${ }^{1,2,5^{*}}$
}

\begin{abstract}
Pembrolizumab monotherapy has become the preferred treatment for patients with advanced non-small cell lung carcinoma (NSCLC) and a programmed cell death-ligand 1 (PD-L1) tumor proportion score (TPS) of at least 50\%. However, little is known about the value of adding chemotherapy to pembrolizumab in this setting. Therefore, we performed an indirect comparison for pembrolizumab plus chemotherapy versus pembrolizumab, using the frequentist methods. The primary outcomes were overall survival (OS), progression-free survival (PFS) and objective response rate (ORR). Data were retrieved from randomized trials comparing pembrolizumab plus chemotherapy or pembrolizumab monotherapy against chemotherapy. Five trials involving 1289 patients were included. Direct meta-analysis showed that both pembrolizumab plus chemotherapy (ORR: relative risk (RR) 2.16; PFS: hazard ratio (HR) 0.36; OS: HR 0.51) and pembrolizumab alone (ORR: RR 1.33; PFS: HR, 0.65; OS: HR 0.67) improved clinical outcomes compared with chemotherapy. Indirect comparison showed that pembrolizumab plus chemotherapy was superior to pembrolizumab alone, in terms of ORR (RR 1.62, 1.18-2.23) and PFS (HR 0.55, 0.32-0.97). A trend towards improved OS was also observed (HR 0.76, $0.51-1.14$ ). In conclusion, the addition of chemotherapy to pembrolizumab further improves the outcomes of patients with advanced NSCLC and a PD-L1 TPS of at least 50\%.
\end{abstract}

Keywords: Non-small cell lung cancer, Programmed cell death-ligand 1, Pembrolizumab, Chemotherapy, First-line

\section{Introduction}

With recent advance of immune checkpoint inhibitor treatment that blocks the PD-1 (programmed cell death 1) and PD-L1 (programmed cell death-ligand 1) pathway, pembrolizumab monotherapy has replaced platinumdoublet chemotherapy as first-line treatment in patients with advanced non-small cell lung carcinoma (NSCLC) and a PD-L1 tumor proportion score (TPS) of $50 \%$ or more [1]. Among patients with unselected PD-L1 expression, pembrolizumab plus chemotherapy is superior to chemotherapy alone [2]. However, whether combination of pembrolizumab and chemotherapy could further improve the clinical outcomes compared with pembrolizumab

\footnotetext{
* Correspondence: hongshd@sysucc.org.cn; zhangli6@mail.sysu.edu.cn ${ }^{\dagger}$ Yixin Zhou, Zuan Lin and Xuanye Zhang contributed equally to this work. ${ }^{1}$ State Key Laboratory of Oncology in South China, Guangzhou, China Full list of author information is available at the end of the article
}

alone remains an urgent controversy due to the lack of head-to-head comparison.

We evaluated the efficacy of pembrolizumab (pem) plus chemotherapy (chemo) versus pembrolizumab alone for the first-line treatment of patients with advanced NSCLC and a PD-L1 TPS of $\geq 50 \%$ using indirect comparison meta-analysis.

\section{Methods}

Study eligibility

We identified eligible randomized controlled trials that compared pembrolizumab plus chemotherapy or pembrolizumab alone with chemotherapy for first-line treatment of advanced NSCLC from Pubmed, Embase and the Cochrane Central Register, with the search terms including pembrolizumab, non-small cell lung cancer, and randomized controlled trial (Additional file 1: Supplemental Methods). The 
abstracts from major conference proceedings of the American Society of Clinical Oncology (ASCO), the European Society of Medical Oncology (ESMO), the American Association for Cancer Research (AACR), and the World Conference on Lung Cancer (WCLC) were also reviewed. Studies were restricted to English language published or presented before November 1, 2018.

\section{Data extraction}

Data were extracted with a predefined information sheet. The primary outcomes for this study were overall survival (OS), progression-free survival (PFS) and objective response rate (ORR). We extracted the hazard ratios (HRs) and their 95\% confidence intervals (CIs) for OS and PFS, and dichotomous data for ORR. Other items included acronym of the trial, number of patients enrolled, and clinicopathological characteristics of the patients.

\section{Data analyses}

Direct comparisons were performed for arm A (pembrolizumab plus chemotherapy) versus arm $\mathrm{C}$ (chemotherapy), and arm B (pembrolizumab) versus arm C (chemotherapy), respectively. The pooled estimates for PFS and OS were presented with HRs, 95\% CIs and $P$ values calculated using the inverse-variance-weighted method, while the measures for dichotomous data (ORR) were pooled with the relative risks (RRs), $95 \%$ CIs and $P$ values using the Mantel Haenszel method. A fixed-effect or random-effect model was adopted depending on between-study heterogeneity.

Indirect comparison was performed for arm A versus arm B, linked by arm C. The adjusted indirect comparison was calculated using the frequentist methods with the following formulas [3]: $\log \mathrm{HR}_{\mathrm{AB}}=\log \mathrm{HR}_{\mathrm{AC}}-\log$ $\mathrm{HR}_{\mathrm{BC}}$, and its standard error (SE) for the log $\mathrm{HR}$ was $S E$ $(\log H R \mathrm{AB})=\sqrt{S E(\log H R \mathrm{AC})^{2}+S E(\log H R \mathrm{BC})^{2}} \cdot \mathrm{RR}$ was calculated similarly as the above formulas. $H R<1$ or $R R>1$ indicates that pembrolizumab plus chemotherapy is superior to pembrolizumab alone, vice versa.

All statistical analyses were conducted using SAS statistical software (version 15.0, SAS Institute Inc). Statistical significance was defined as a 2 -sided $P<.05$.

\section{Results}

A total of five trials involving 1289 patients were included (trial selection process shown in Additional file 1: Figure S1) [1, 4-7]. The assessment of risk of bias is presented in Additional file 1: Table S1.

The main characteristics and outcomes of the included trials are summarized in Table 1 . Three trials investigated pembrolizumab plus chemotherapy versus chemotherapy and two trials investigated pembrolizumab alone versus chemotherapy. All the trials used the 22C3
pharmDx assay (Agilent Technologies) to assess PD-L1 expression with immunohistochemical method. All the included trials used standard-of-care chemotherapeutic regimens according to practice guidelines. The median follow-up time ranged from 7.8 months to 23.9 months. All the five trials provided ORR data; OS and PFS data were not reported in KEYNOTE-021 trial cohort G [4].

\section{Direct meta-analysis}

Significant difference of ORR was observed in favor of pembrolizumab plus chemotherapy versus chemotherapy $\left(\mathrm{RR}_{\mathrm{pem}+\text { chemo/chemo }} 2.16,95 \%\right.$ CI 1.66-2.82; $P<0.001$; heterogeneity, $P=0.441$ ). And for pembrolizumab vs chemotherapy, the pooled $\mathrm{RR}_{\text {pem/chemo }}$ was 1.33 (95\% CI 1.11-1.58; $P=0.002$; heterogeneity, $P=0.260$ ) (Fig. 1a).

For PFS, pembrolizumab plus chemotherapy significantly reduced the risk of disease progression compared with chemotherapy $\left(\mathrm{HR}_{\mathrm{pem}+\text { chemo/chemo }}, 0.36 ; 95 \% \mathrm{CI}\right.$ $0.27-0.48 ; \mathrm{z}=7.03, P<0.001$; heterogeneity, $P=0.925)$. While pembrolizumab monotherapy failed to demonstrate significant improvement in PFS $\left(\mathrm{HR}_{\mathrm{pem} / \mathrm{chemo}}\right.$, 0.65 ; 95\% CI $0.40-1.04 ; \mathrm{z}=1.82, P=0.069$; heterogeneity, $P=0.009$ ) (Fig. 1b).

In terms of OS, both pembrolizumab plus chemotherapy $\left(\mathrm{HR}_{\mathrm{pem}+\text { chemo/chemo }}, 0.51 ; 95 \%\right.$ CI $0.35-0.72 ; \mathrm{z}=$ 3.71, $P<0.001)$ and pembrolizumab monotherapy $\left(\mathrm{HR}_{\text {pem } / \text { chemo }}, 0.67 ; 95 \%\right.$ CI $0.56-0.80 ; \mathrm{z}=4.57, P<$ $0.001)$ significantly decreased the risk of death compared with chemotherapy (Fig. 1c).

\section{Indirect meta-analysis}

Figure $1 \mathrm{~d}$ showed the relationship of the indirect comparisons. The results indicated that patients treated with pembrolizumab plus chemotherapy had better clinical outcomes including ORR $\left(\mathrm{RR}_{\text {pem }+ \text { chemo/pem }} 1.62,95 \% \mathrm{CI}\right.$

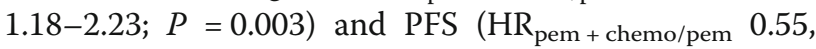
95\% CI 0.32-0.97; $P=0.037$ ) than those treated with pembrolizumab alone. However, there was only a trend towards improved OS with the three-drug combination therapy $\left(\mathrm{HR}_{\mathrm{pem}+\text { chemo/pem }} 0.76,95 \% \mathrm{CI} 0.51-1.14 ; P=\right.$ $0.184)$.

\section{Discussion}

In this hypothesis-generating meta-analysis, we found that pembrolizumab plus chemotherapy is superior to pembrolizumab alone for first-line treatment of patients with advanced NSCLC and a PD-L1 TPS of $\geq 50 \%$, in terms of ORR and PFS. A trend towards improved OS is also observed in the three-drug combination group.

PD-L1 is an established biomarker for selecting patients for first-line treatment with pembrolizumab monotherapy [1]. Although it may be tempting to believe that pembrolizumab monotherapy attains a better toxicity profile while retaining survival benefit in patients with a PD-L1 TPS of 


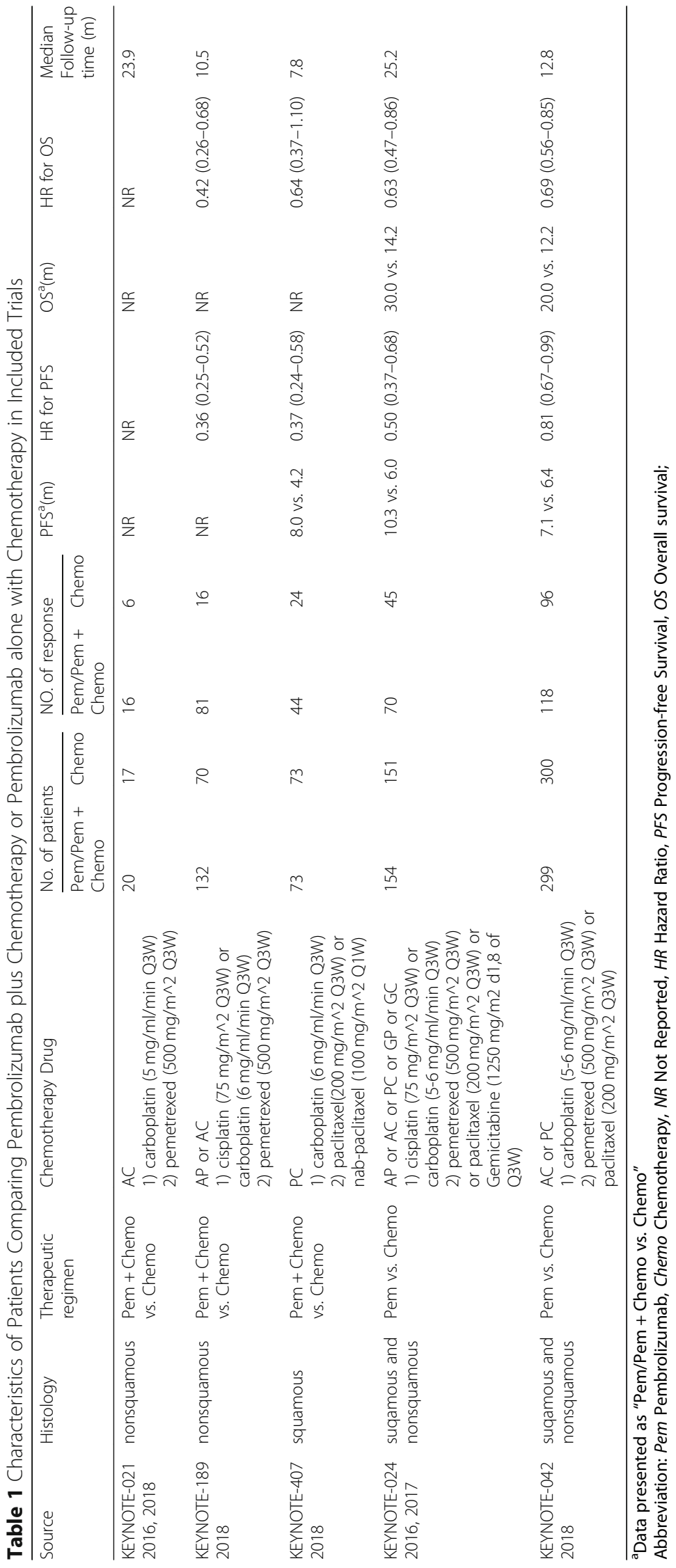

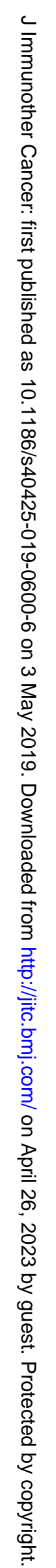



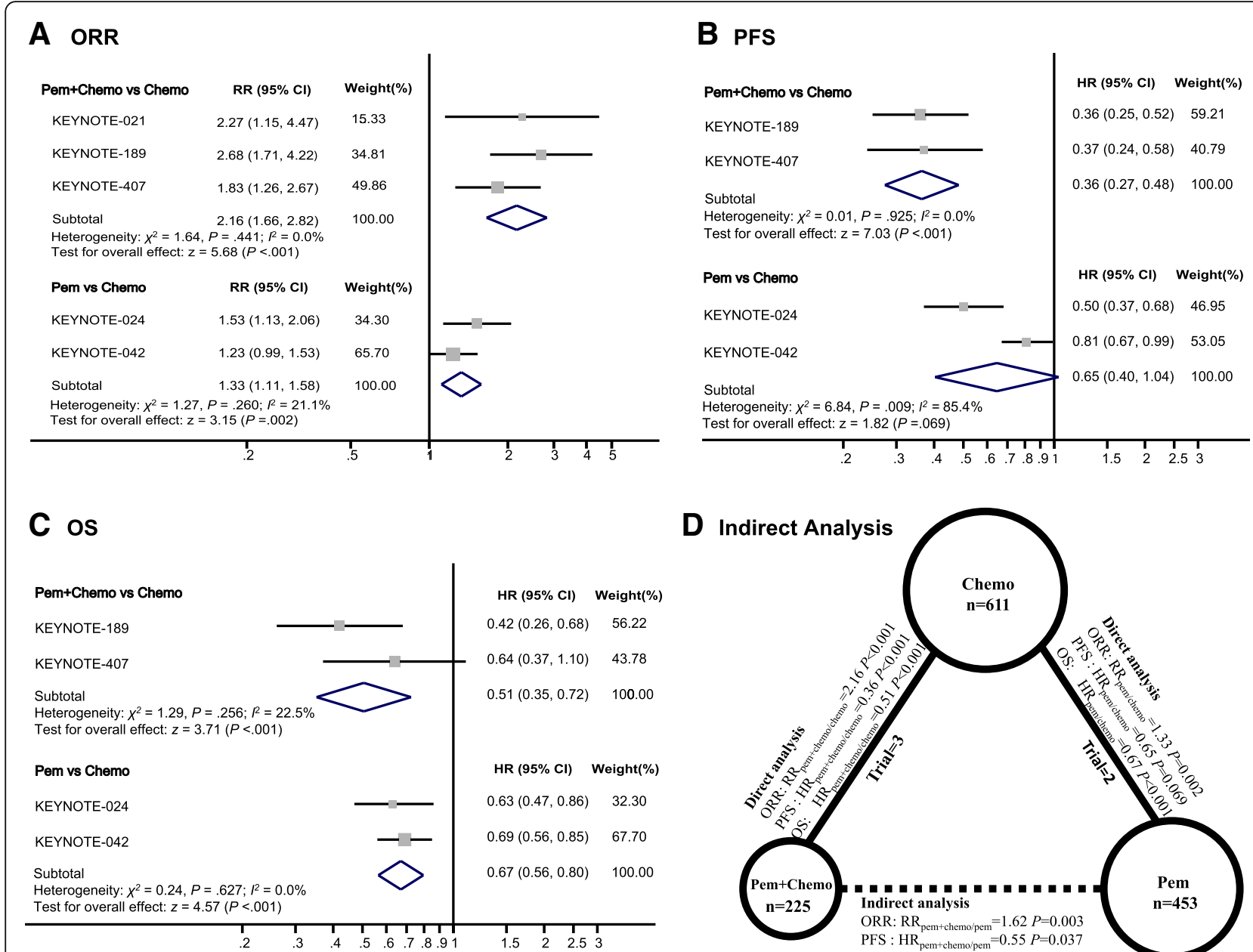

D Indirect Analysis

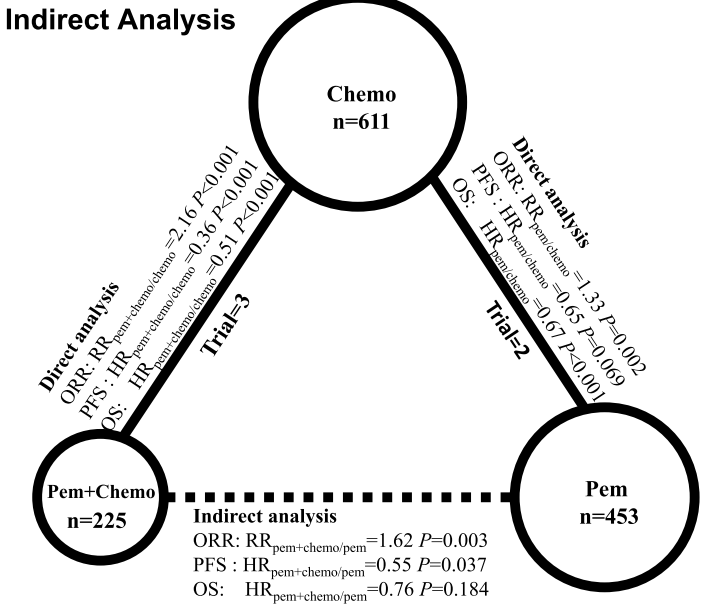

Fig. 1 Direct Comparisons between Pembrolizumab plus Chemotherapy or Pembrolizumab Alone with Chemotherapy and Indirect Comparison between Pembrolizumab plus Chemotherapy versus Pembrolizumab Alone. $\mathbf{a}$, b and $\mathbf{c}$ showed the Forest plot of risk ratios (RRs) and hazard ratios (HRs) directly comparing objective response rate (a), progression-free survival (b), and overall survival (c) between pembrolizumab plus chemotherapy or pembrolizumab alone with chemotherapy. The size of the data markers (squares) corresponds to the weight of the study in the meta-analysis. The horizontal line crossing the square represents the $95 \% \mathrm{Cl}$. The diamonds represent the estimated overall effect, based on the meta-analysis. In $\mathbf{d}$, solid lines represented the existence of direct comparisons between treatment regimens, and dashed line represented the indirect comparison between pem + chemo versus pem. The size of the circle corresponds to the enrolled patient number. All statistical tests were 2-sided. Abbreviations: Pem Pembrolizumab, Chemo Chemotherapy

at least $50 \%$. The challenge is that less than $50 \%$ of patients with advanced NSCLC ever receive second-line therapy due to rapid deterioration during disease progression [8]. Therefore, maximizing the chance of response to first-line treatment and delaying the occurrence of drug resistance is clinically relevant. Another challenge is the intratumoral heterogeneity of PD-L1 expression [9]. A fine-needle aspiration specimen does not represent the whole picture of the tumour and high PD-L1 expression detected in this circumstance might be "false positive". Additionally, the cutoff value of $50 \%$ is not ideal for benefit stratification. A retrospective study found that pembrolizumab only produced moderate efficacy in patients with a PD-L1 TPS of 50-74\% (ORR 21.6\%; PFS 3.2 months; OS 20.6 months) or $50-89 \%$ (ORR 25.2\%; PFS 3.7 months; OS
15.2 months) [10], indicating that the exact beneficial population might be those with even higher PD-L1 level, though the optimal cutoff remains not illustrated. These challenges probably explained the phenomenon that pembrolizumab monotherapy only produces a response rate of $40-45 \%$ and that the separation of survival curves is in a delayed manner $[5,7]$.

Our pooled analysis indicates that pembrolizumab monotherapy did not significantly improved PFS compared with chemotherapy while pembrolizumab plus chemotherapy outperforms chemotherapy in terms of all the tested outcomes including ORR, PFS and OS. Indirect comparison shows that the addition of chemotherapy to pembrolizumab further increases the chance of response by $62 \%$. Additionally, the risk of disease progression and 
death is reduced by 45 and $24 \%$, respectively. Although the improvement of OS with the three-drug combination versus pembrolizumab single agent is not statistically significant, it is likely due to the short duration of follow-up in KEYNOTE-407 trial [6]. An update analysis with extended follow-up will be needed. Our findings lend support for the hypothesis that chemotherapeutic agents may exert immune-potentiating effects under certain circumstance. Based on these data, it may be reasonable to recommend that patients with high tumor volume to be treated with the combinatorial therapy to produce deeper and longer response, while patients with low tumor volume or with very high PD-L1 TPS to be treated with pembrolizumab alone.

A strength of this work is the quality of evidence available and used in the meta-analysis. Source data were obtained from five well-designed randomised controlled trials involving over 1000 patients. The experimental drug and methods for PD-L1 expression is the same. Thus, the meta-analysis could overcome the problem of inadequate power of each individual trial by pooling data together and minimize between-study heterogeneity. Albeit the strength above, we encountered several limitations during this study. First of all, our meta-analysis relies on published results rather than on individual patients' data. Secondly, we lacked data from head-to-head comparison. Finally, the data from pembrolizumab plus chemotherapy are retrieved from subgroup analyses. Therefore, the interpretation of the results needs additional caution. However, there was no important difference between trials with pembrolizumab plus chemotherapy and trials with pembrolizumab monotherapy included for the analyses, which makes the indirect comparison reliable to some extent. Given these limitations, head-to-head randomized trials will be required to directly compare pembrolizumab plus chemotherapy against pembrolizumab alone. Future researches should also explore the optimal cutoff value of PD-L1 above which pembrolizumab is non-inferior to pembrolizumab plus chemotherapy.

In conclusion, the addition of chemotherapy to pembrolizumab as first-line treatment further improves the outcomes of patients with advanced NSCLC and a PD-L1 TPS of at least 50\%. With proved survival benefit, manageable toxicities and avoidance of PD-L1-based patient selection, clinicians could prefer pembrolizumab plus chemotherapy in patients without contraindications, especially for those with high tumor burden.

\section{Additional file}

Additional file 1: Supplemental Methods. Search strategies and number of studies yielded from each database. Table S1. Quality assessment: risk of bias by Cochrane Collaboration's tool. Figure S1. Trial Selection Process (PDF $337 \mathrm{~kb}$ )

\section{Abbreviations}

AACR: American Association for Cancer Research; advanced NSCLC: Advanced non-small cell lung carcinoma; ASCO: American Society of Clinical Oncology; Chemo: Chemotherapy; Cl: Confidence interval; ESMO: European Society of Medical Oncology; HR: Hazard ratio; ORR: Objective response rate; OS: Overall survival; PD-1: Programmed cell death 1; PD-L1: Programmed cell death-ligand 1; Pem: Pembrolizumab; PFS: Progression-free survival; RR: Relative risk; SE: Standard error; TPS: Tumor proportion score tumor proportion score; WCLC: World Conference on Lung Cancer

\section{Acknowledgments}

We sincerely appreciate Yidu Cloud Corporation (Beijing, China) for data management.

\section{Funding}

This study was funded by grants 2016 YFC0905500 and 2016 YFC0905503 from the National Key R\&D Program of China; 81602005, 81702283, and 81602011 from the National Natural Science Funds of China; 16zxyc04 from the Outstanding Young Talents Program of Sun Yat-sen University Cancer Center; $17 y k p y 81$ from the Central Basic Scientific Research Fund for Colleges-Young Teacher Training Program of Sun Yat-sen University; 2017B020227001 from the Science and Technology Program of Guangdong Province. The funding sources had no role in the design and conduct of the study; collection, management, analysis, and interpretation of the data; preparation, review, or approval of the manuscript; and decision to submit the manuscript for publication.

Availability of data and materials

All data generated or analysed during this study are included in the published article.

\section{Authors' contributions}

YZ, ZL and XZ contributed to data acquisition, data interpretation, and statistical analysis and drafting of the manuscript. SH and LZ contributed to the study design, data acquisition, data interpretation, statistical analysis. All the authors contributed to critical revision of the manuscript.

Ethics approval and consent to participate

Not applicable.

Consent for publication

Not applicable.

\section{Competing interests}

Li Zhang has received research support from AstraZeneca, Eli Lilly and company, and Roche. No other disclosures were reported.

\section{Publisher's Note}

Springer Nature remains neutral with regard to jurisdictional claims in published maps and institutional affiliations.

\section{Author details}

${ }^{1}$ State Key Laboratory of Oncology in South China, Guangzhou, China. ${ }^{2}$ Collaborative Innovation Center for Cancer Medicine, Guangzhou, China. ${ }^{3}$ Department of VIP region, Sun Yat-sen University Cancer Center, Guangzhou, China. ${ }^{4}$ Department of Clinical Research, Sun Yat-sen University Cancer Center, Guangzhou, China. ${ }^{5}$ Department of Medical Oncology, Sun Yat-sen University Cancer Center, 651 Dongfeng East Road, Guangzhou 510060, China. ${ }^{6}$ Department of Radiotherapy, Sun Yat-sen University Cancer Center, Guangzhou, China.

Received: 2 January 2019 Accepted: 23 April 2019

Published online: 03 May 2019

\section{References}

1. Reck M, Rodriguez-Abreu D, Robinson AG, Hui R, Csoszi T, Fulop A, et al. Pembrolizumab versus chemotherapy for PD-L1-positive non-small-cell lung Cancer. N Engl J Med. 2016;375:1823-33.

2. Zhou Y, Chen C, Zhang X, Fu S, Xue C, Ma Y, et al. Immune-checkpoint inhibitor plus chemotherapy versus conventional chemotherapy for first-line 
treatment in advanced non-small cell lung carcinoma: a systematic review and meta-analysis. J Immunother Cancer. 2018;6:155-65.

3. Bucher HC, Guyatt GH, Griffith LE, Walter SD. The results of direct and indirect treatment comparisons in meta-analysis of randomized controlled trials. J Clin Epidemiol. 1997;50:683-91.

4. Langer CJ, Gadgeel SM, Borghaei H, Papadimitrakopoulou VA, Patnaik A, Powell SF, et al. Carboplatin and pemetrexed with or without pembrolizumab for advanced, non-squamous non-small-cell lung cancer: a randomised, phase 2 cohort of the open-label KEYNOTE-021 study. Lancet Oncol. 2016;17:1497-508.

5. Gandhi L, Rodriguez-Abreu D, Gadgeel S, Esteban E, Felip E, De Angelis F, et al. Pembrolizumab plus chemotherapy in metastatic non-small-cell lung Cancer. N Engl J Med. 2018;378:2078-92.

6. Paz-Ares L, Luft A, Vicente D, Tafreshi A, Gumus M, Mazieres J, et al. Pembrolizumab plus chemotherapy for squamous non-small-cell lung Cancer. N Engl J Med. 2018;379:2040-51.

7. Lopes G, Wu Y-L, Kudaba I, Kowalski D, Cho BC, Castro G, et al. Pembrolizumab (pembro) versus platinum-based chemotherapy (chemo) as first-line therapy for advanced/metastatic NSCLC with a PD-L1 tumor proportion score (TPS) $\geq 1 \%$ : Open-label, phase 3 KEYNOTE-042 study. J Clin Oncol. 2018;36:LBA4-LBA. https://doi.org/10.1200/JCO.2018.36.18_suppl.LBA4.

8. Davies J, Patel M, Gridelli C, de Marinis F, Waterkamp D, McCusker ME. Realworld treatment patterns for patients receiving second-line and third-line treatment for advanced non-small cell lung cancer: a systematic review of recently published studies. PLoS One. 2017;12:e0175679.

9. Pinato DJ, Shiner RJ, White SD, Black JR, Trivedi P, Stebbing J, et al. Intratumoral heterogeneity in the expression of programmed-death (PD) ligands in isogeneic primary and metastatic lung cancer: implications for immunotherapy. Oncoimmunology. 2016;5:e1213934.

10. Jimenez Alguilar E, Gainor J, Kravets S, Khosrowjerdi S, Lydon C, Adeni A, et al. MA04.05 outcomes in NSCLC patients treated with first-line Pembrolizumab and a PD-L1 TPS of $50-74 \%$ vs $75-100 \%$ or $50-89 \%$ vs $90-$ 100\%. J Thorac Oncol. 2018:13:S367-S8.

Ready to submit your research? Choose BMC and benefit from:

- fast, convenient online submission

- thorough peer review by experienced researchers in your field

- rapid publication on acceptance

- support for research data, including large and complex data types

- gold Open Access which fosters wider collaboration and increased citations

- maximum visibility for your research: over $100 \mathrm{M}$ website views per year

At $\mathrm{BMC}$, research is always in progress.

Learn more biomedcentral.com/submissions 